\title{
Treadmill exercise ameliorates memory impairment through ERK-Akt-CREB-BDNF signaling pathway in cerebral ischemia gerbils
}

\author{
Su-Shin Lee', Chang-Ju Kim', Mal-Soon Shin², Baek-Vin Lim** \\ 1Department of Physiology, College of Medicine, Kyung Hee University, Seoul, Korea \\ ${ }^{2}$ School of Global Sport Studies, Korea University, Sejong, Korea \\ ${ }^{3}$ Division of Leisure \& Sports Science, Department of Exercise Prescription, Dongseo University, Busan, Korea
}

Neuronal cell death in the hippocampus by cerebral ischemia causes disability of memory function. Cerebral ischemia also alters the expressions of brain-derived neurotrophic factor (BDNF), cyclic adenosine monophosphate-responsive element binding protein (CREB), extracellular signal-regulated protein kinase (ERK), and phosphatidylinositol 3-kinase/protein kinase B (Akt). In the present study, we investigated the effect of treadmill exercise on cerebral ischemia in relation with ERK-Akt-CREB-BDNF signaling pathway in the hippocampus using gerbils. Induction of cerebral ischemia deteriorated short-term memory with suppression of phosphorylation of ERK-Akt-CREB-BDNF pathway in the hippocampus of gerbils. Enhancement of apoptosis in the hippo- campus was accompanied in the ischemia gerbils. Treadmill exercise improved short-term memory through enhancing phosphorylation of ERK-Akt-CREB-BDNF pathway with suppressing apoptosis in the hippocampus of the ischemia gerbils. The present results suggest that improvement of memory function after cerebral ischemia by treadmill exercise may be involved in the ERK-Akt-CREB-BDNF signaling pathway, resulting in inhibition of apoptosis in the hippocampus.

Keywords: Treadmill exercise, Cerebral ischemia, Short-term memory, Extracellular signal-regulated protein kinase, Brain-derived neurotrophic factor, Apoptosis

\section{INTRODUCTION}

Neuronal cell death in the hippocampus by cerebral ischemia causes disability of memory function. Cerebral ischemia also alters the expressions of brain-derived neurotrophic factor (BDNF), cyclic adenosine monophosphate-responsive element binding protein (CREB), extracellular signal-regulated protein kinase (ERK), and phosphatidylinositol 3-kinase (PI3-kinase)/protein kinase $\mathrm{B}$ (Akt) (Irving and Bamford, 2002; Ko et al., 2018; Wang et al., 2009; Zhu et al., 2004).

BDNF is a member of the neurotrophic family involved in neuronal survival and differentiation, and it plays an important role in learning process due to its involvement in long-term potentiation in the hippocampus (Yamada et al., 2002). BDNF is a CREB target gene and plays a crucial role in the synaptic plasticity of the brain (Poo, 2001). BDNF is involved in neuronal survival and activates ERK, CREB, and Akt after binding to its receptor tyrosine kinase B (TrkB) (Numakawa et al., 2010). Activation of TrkB for BDNF triggers several parallel signaling pathways, including the Ras/ERK, PI3-kinase (Santos et al., 2010). BDNF is known to exert protective effect against ischemic insult (Ko et al., 2018).

CREB-mediated transcription system is necessary for the induction of ischemic tolerance after global ischemia (Hara et al., 2003). CREB is associated with neuronal survival, memory consolidation, and synaptic plasticity (Impey et al., 2004). CREB activation is a critical event in neuroprotection against ischemic injury (Meller et al., 2005).

ERK is one of the mitogen-activated protein kinase families
${ }^{*}$ Corresponding author: Baek-Vin Lim (iD https://orcid.org/0000-0003-4017-573X Division of Leisure \& Sports Science, Department of Exercise Prescription,

Dongseo University, 47 Jurye-ro, Sasang-gu, Busan 47011, Korea

E-mail: todd64@naver.com

Received: December 5, 2019 / Accepted: January 12, 2020
This is an Open Access article distributed under the terms of the Creative Commons Attribution Non-Commercial License (https://creativecommons.org/licenses/by-nc/4.0/) which permits unrestricted non-commercial use, distribution, and reproduction in any medium, provided the original work is properly cited. 
and modulates cell growth, differentiation, and survival. Reduced ERK signaling contributes to neuronal cell death in hippocampal dentate after transient cerebral ischemia (Yan et al., 2007). BDNF/ TrkB activates ERK-regulated CREB phosphorylation (Whitfield et al., 2011).

Apoptosis after cerebral ischemia is one of the major pathways that lead to the process of cell death (Mattson et al., 2001). Two important groups of proteins involved in ischemic apoptotic cell death are the members of the Bcl-2 family and a class of cysteine proteases known as caspases (Choi et al., 2017; Park et al., 2016). $\mathrm{Bcl}-2$ family is classified into two functionally distinct groups: Bcl-2, an anti-apoptotic protein, and Bax, a pro-apoptotic protein. Caspase- 3 is one of the most widely studied members of the caspase family, and it is one of the key executors of apoptosis.

Memory improving and antiapoptotic effects of exercise are well documented (Ko et al., 2018; Lee et al., 2019). In the present study, we investigated the effect of treadmill exercise on cerebral ischemia in relation with ERK-Akt-CREB-BDNF signaling pathway in the hippocampus using gerbils.

\section{MATERIALS AND METHODS}

\section{Experimental animals}

Male Mongolian gerbils weighing $60 \pm 10 \mathrm{~g}$ were used in this experiment. The experimental procedures were performed in accordance with the animal care guidelines of the National Institutes of Health and the Korean Academy of Medical Sciences. The gerbils were randomly assigned into four groups ( $\mathrm{n}=10$ in each group): the sham-operation group, the sham-operation and exercise group, the ischemia-induction group, and the ischemia-induction and exercise group.

\section{Induction of transient global ischemia}

Transient global ischemia was induced according to the previously described surgical procedure (Choi et al., 2017; Lee et al., 2019). The gerbils were anesthetized with Zoletil $50(10 \mathrm{mg} / \mathrm{kg}$, intraperitoneally; Vibac Laboratories, Carros, France). Following bilateral neck incision, both common carotid arteries were exposed and occluded with aneurysm clips for $5 \mathrm{~min}$. The clips were then removed to restore cerebral blood flow. Body and rectal temperature were maintained at $36^{\circ} \mathrm{C} \pm 0.5^{\circ} \mathrm{C}$ during surgery using Homeothermic Blanket Control Unit (Harvard Apparatus, Massachusetts, MA, USA).

\section{Treadmill exercise protocol}

The gerbils in the treadmill exercise groups were subjected to run on a treadmill for 30 min once a day for 14 days, starting one day after induction of cerebral ischemia. The exercise load consisted of running at a speed of $2 \mathrm{~m} / \mathrm{min}$ for the first $5 \mathrm{~min}, 3 \mathrm{~m} / \mathrm{min}$ for the next $5 \mathrm{~min}$, and $4 \mathrm{~m} / \mathrm{min}$ for the last $20 \mathrm{~min}$ with the $0^{\circ}$ inclination.

\section{Step-down avoidance task}

In order to evaluate short-term memory in the gerbils, the latency in the step-down avoidance task was determined as the previously described method (Kim et al., 2019; Ko et al., 2019). On the 12th day starting treadmill exercise, the gerbils were trained in a step-down avoidance test. The gerbils were positioned on a $7 \times 25-\mathrm{cm}$ platform with a height of $2.5 \mathrm{~cm}$, and then allowed to rest on the platform for $2 \mathrm{~min}$. The platform faced a $42 \times 25-\mathrm{cm}$ grid of parallel $0.1-\mathrm{cm}$-caliber stainless steel bars, which were spaced $1 \mathrm{~cm}$ apart. In the training sessions, the animals received a $0.5-\mathrm{mA}$ scramble foot shock for $3 \mathrm{sec}$ immediately upon stepping down. Retention time was assessed 1 day after training session. The interval for gerbils stepping down and placing all four paws on the grid was defined as the latency in the step-down avoidance task. The latency over $300 \mathrm{sec}$ was counted as $300 \mathrm{sec}$.

\section{Tissue preparation}

For brain tissue preparation, the animals were fully anesthetized with Zoletil $50(10 \mathrm{mg} / \mathrm{kg}$, intraperitoneally; Vibac Laboratories, Carros, France), transcardially perfused with $50 \mathrm{mM}$ phosphate-buffered saline, and fixed with freshly prepared solution consisting of $4 \%$ paraformaldehyde in $100 \mathrm{mM}$ phosphate buffer $(\mathrm{pH}, 7.4)$. Brains were then removed, postfixed in the same fixative overnight, and transferred into a 30\% sucrose solution for cryoprotection. Coronal sections of $40-\mu \mathrm{m}$ thickness were made using a freezing microtome (Leica, Nussloch, Germany).

\section{Caspase-3 immunohistochemistry}

Caspase-3 immunohistochemistry was performed according to a previously described method (Park et al., 2019; Song et al., 2018). The sections were incubated overnight with mouse anti-caspase-3 antibody (1:500; Santa Cruz Biotechnology, Santa Cruz, CA, USA) and then they were incubated for another $1 \mathrm{hr}$ with the biotinylated mouse secondary antibody. The bound secondary antibody was then amplified using a Vector Elite ABC kit (1:200; Vector Laboratories, Burlingame, CA, USA). The antibody-biotin-avidin-peroxidase complex was visualized using $0.02 \%$ diaminobenzidine. 
The sections were finally mounted onto gelatin-coated slides. The slides were air-dried overnight at room temperature, and the coverslips were mounted using Permount (Fisher Scientific, Pittsburgh, PA, USA).

\section{Western blot analysis}

Western blot analysis was performed, as the previously described method (Kim et al., 2019; Park et al., 2019; Song et al., 2018). Brain tissues were lysed in an ice-cold whole cell lysate buffer. Protein of $30 \mu \mathrm{g}$ was separated on sodium dodecyl sulfate-polyacrylamide gels and transferred onto a nitrocellulose membrane (Whatman, Clifton, NJ, USA). The nitrocellulose membrane was blocked by incubation for $90 \mathrm{~min}$ at room temperature in tris-buffered saline containing $0.1 \%$ tween 20 and 5\% nonfat dried milk (Becton Dickinson, Sparks, MD, USA). Rabbit glyceraldehyde 3-phosphate dehydrogenase (GAPDH) antibody (1:10,000; Affinity Bioreagent, Rockford, IL, USA), rabbit BDNF, TrkB, and phosphorylatedPI3-kinase (p-PI3-kinase) antibody (1:1,000; Santa Cruz Biotechnology), rabbit Akt and phosphorylated-Akt (p-Akt) antibody (1:1,000; Cell Signaling Technology, Beverly, MA, USA), mouse Bax, Bcl-2, PI3-kinase, ERK and phosphorylated-ERK (p-ERK) antibody (1:1,000; Santa Cruz Biotechnology), rabbit CREB (1: 1,000; Millipore, Billerica, MA, USA), and goat phosphorylatedCREB antibody (1:1,000; Santa Cruz Biotechnology) were used as the primary antibodies. Horseradish peroxidase-conjugated antirabbit antibody for GAPDH, BDNF, TrkB, CREB, p-PI3-kinase, Akt, and p-Akt (1:3,000; Vector Laboratories), horseradish peroxidase-conjugated anti-mouse antibody (1:3,000; Vector Laboratories) for Bax, Bcl-2, PI3-kinase, ERK and p-ERK, and horseradish peroxidase-conjugated anti-goat antibody (1:5,000; Amersham Pharmacia Biotechnology GmbH, Freiburg, Germany) for p-CREB were used as the secondary antibodies. Experiments were performed under normal laboratory conditions and at room temperature, except for the transfer to membranes. The transfer was performed at $4^{\circ} \mathrm{C}$ with a cold pack and prechilled buffer. Band detection was performed using enhanced chemiluminescence detection kits (Santa Cruz Biotechnology).

\section{Data analysis}

The area of the hippocampal CA1 region was measured using Image-Pro Plus software (Media Cybernetics, Silver Spring, MD, USA). The numbers of caspase-3-positive cells in the hippocampus were counted hemilaterally and the data are expressed as the number of cells per $\mathrm{mm} 2$ of the hippocampus. To compare the relative expressions of BDNF, TrkB, ERK, p-ERK, Akt, p-Akt,
CREB, p-CREB, Bax, and Bcl-2, the detected bands were calculated densitometrically using Image-Pro Plus software (Media Cybernetics). Statistical analysis was performed using one-way analysis of variance followed by Duncan post boc test. The results are presented as the mean \pm standard error of the mean. Significance was set as $P<0.05$.

\section{RESULTS}

\section{Short-term memory}

The results of the step-through avoidance task are presented in Fig. 1. Short-term memory was impaired by ischemia induction. Treadmill exercise ameliorated ischemia-induced memory impairment.

\section{Expression of BDNF and TrkB in the hippocampus}

The results of the relative expression of BDNF and TrkB in the hippocampus are presented in Fig. 2. BDNF and TrkB expression were decreased by ischemia induction. Treadmill exercise increased BDNF and TrkB expression in the ischemia gerbils.

\section{Expression of CREB and p-CREB in the hippocampus}

The results of the relative expression of CREB and p-CREB in the hippocampus are presented in Fig. 3. p-CREB expression was decreased by ischemia induction, resulting in decrease of p-CREB/CREP ratio. Treadmill exercise increased p-CREB expression in the ischemia gerbils, resulting in increase of $\mathrm{p}-\mathrm{CREB} /$ CREP ratio

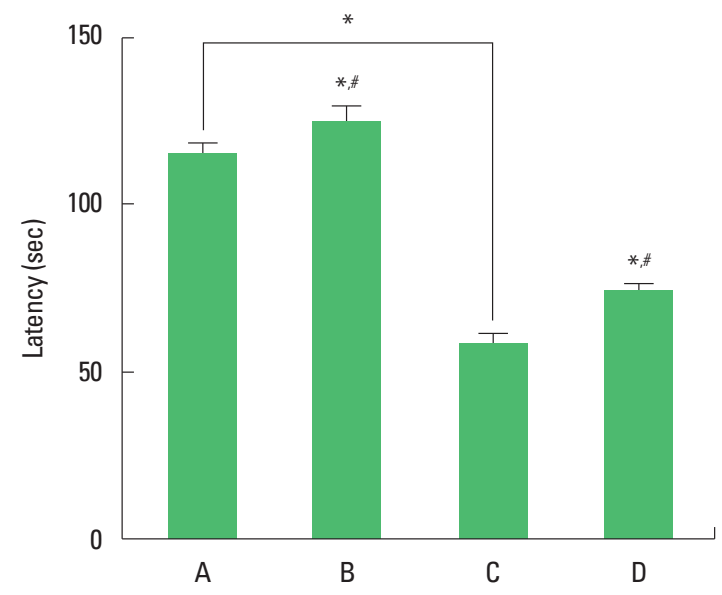

Fig. 1. Latency in the step-down avoidance task. A, sham-operation group; $B$, sham-operation and exercise group; $C$, ischemia-induction group; $D$, ischemia-induction and exercise group. ${ }^{*} P<0.05$ compared to the sham-operation group. ${ }^{*} P<0.05$ compared to the ischemia-induction group. 

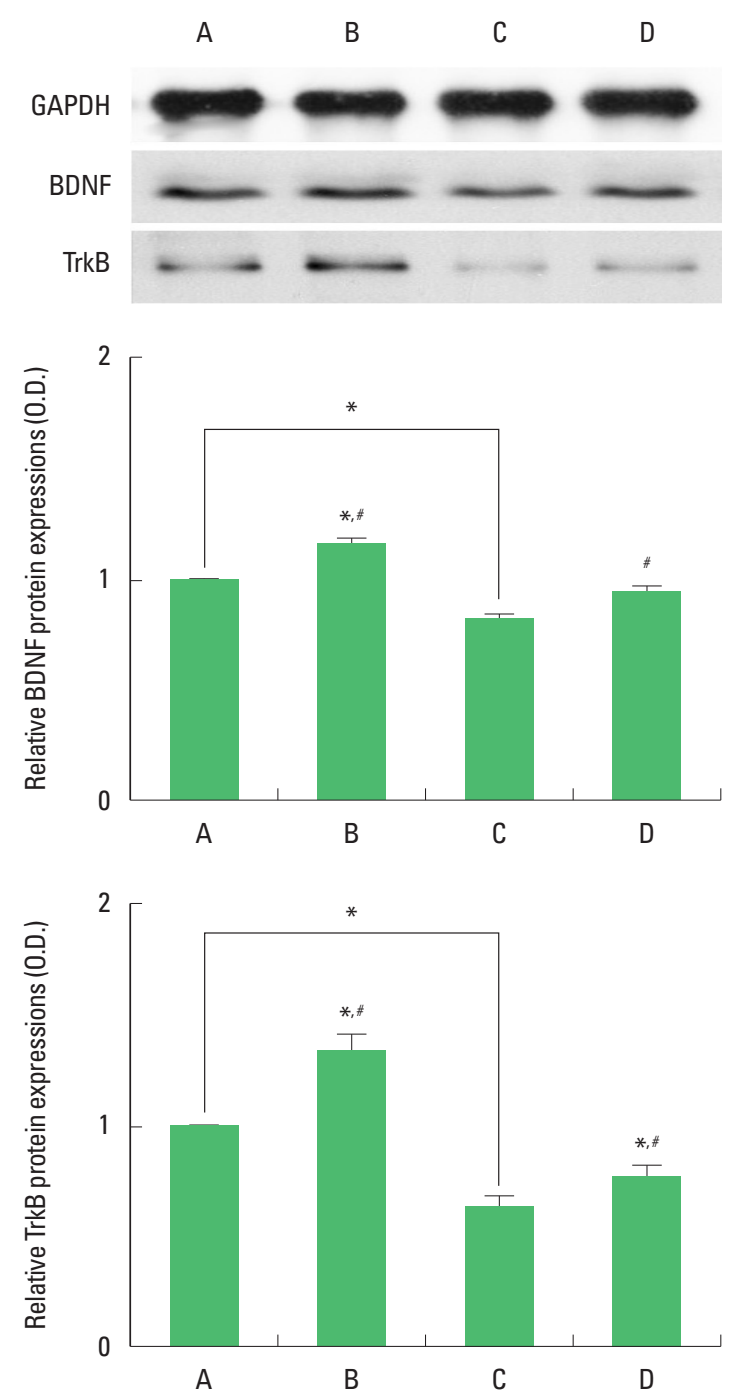

Fig. 2. Brain-derived neurotrophic factor (BDNF) and tyrosine kinase B (TrkB) expression in the hippocampus. Upper panel: representative expression of BDNF and TrkBn. Middle and lower panel: relative expressions of BDNF and TrKB. A, sham-operation group; $B$, sham-operation and exercise group; $C$, ischemia-induction group; $\mathrm{D}$, ischemia-induction and exercise group. ${ }^{*} P<0.05$ compared to the sham-operation group. ${ }^{*} P<0.05$ compared to the ischemia-induction group.

\section{Expression of ERK and p-ERK in the hippocampus}

The results of the relative expression of ERK and p-ERK in the hippocampus are presented in Fig. 4. p-ERK expression was decreased by ischemia induction, resulting in decrease of p-ERK/ ERK ratio. Treadmill exercise increased p-ERK expression in the ischemia gerbils, resulting in increase of p-ERK/ERK ratio.

\section{Expression of Akt and p-Akt in the hippocampus}

The results of the relative expression of Akt and p-Akt in the
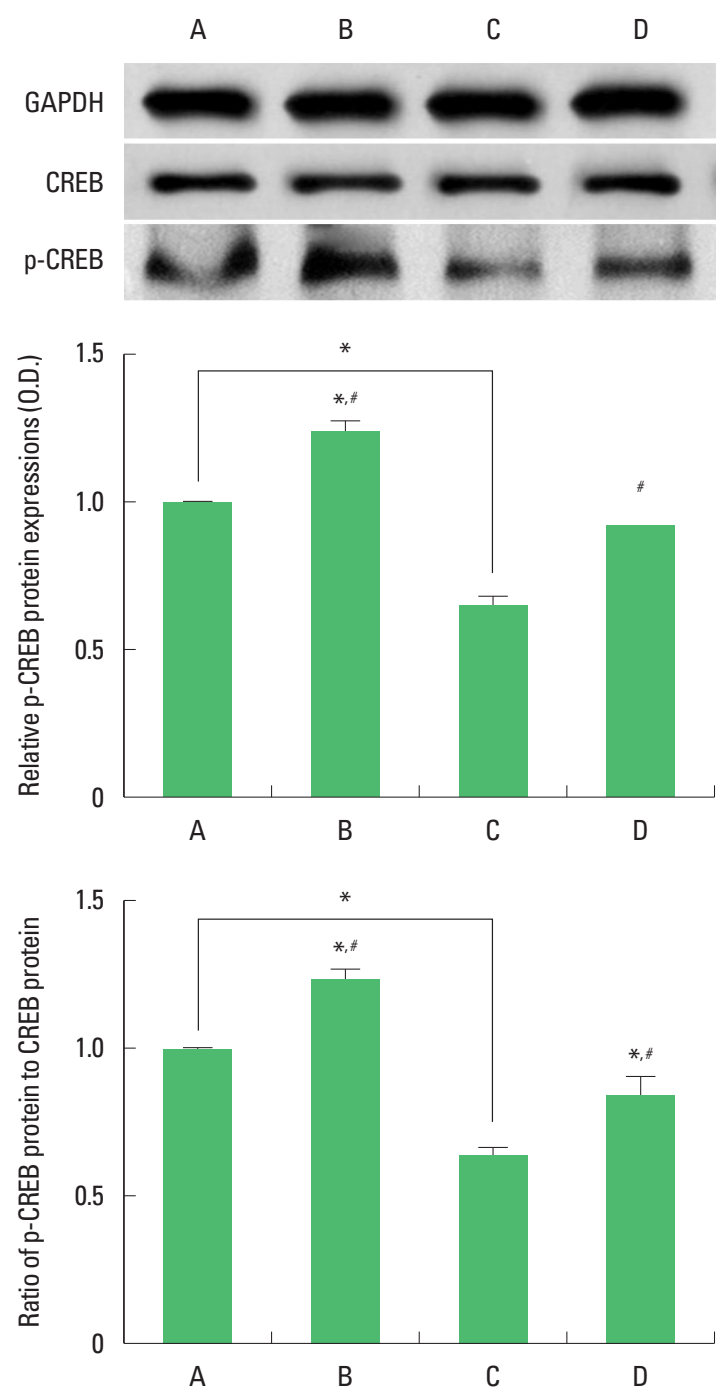

Fig. 3. Cyclic adenosine monophosphate-responsive element binding protein (CREB) and phosphorylated CREP ( $p$-CREB) expression in the hippocampus. Upper panel: representative expression of CREP and p-CREB. Middle and lower panel: relative expressions of CREP and $p$-CREB. $A$, sham-operation group; $B$, sham-operation and exercise group; $C$, ischemia-induction group; $D$, ischemia-induction and exercise group. ${ }^{*} P<0.05$ compared to the sham-operation group. ${ }^{*} P<0.05$ compared to the ischemia-induction group.

hippocampus are presented in Fig. 5. p-Akt expression was decreased by ischemia induction, resulting in decrease of $\mathrm{p}-\mathrm{Akt} / \mathrm{Akt}$ ratio. Treadmill exercise increased $\mathrm{p}-\mathrm{Akt}$ expression in the ischemia gerbils, resulting in increase of $\mathrm{p}-\mathrm{Akt} / \mathrm{Akt}$ ratio.

\section{Expression of PI3-kinase and p-PI3-kinase in the hippocampus}

The results of the relative expression of PI3-kinase and p-PI3-kinase in the hippocampus are presented in Fig. 6. p-PI3-kinase ex- 

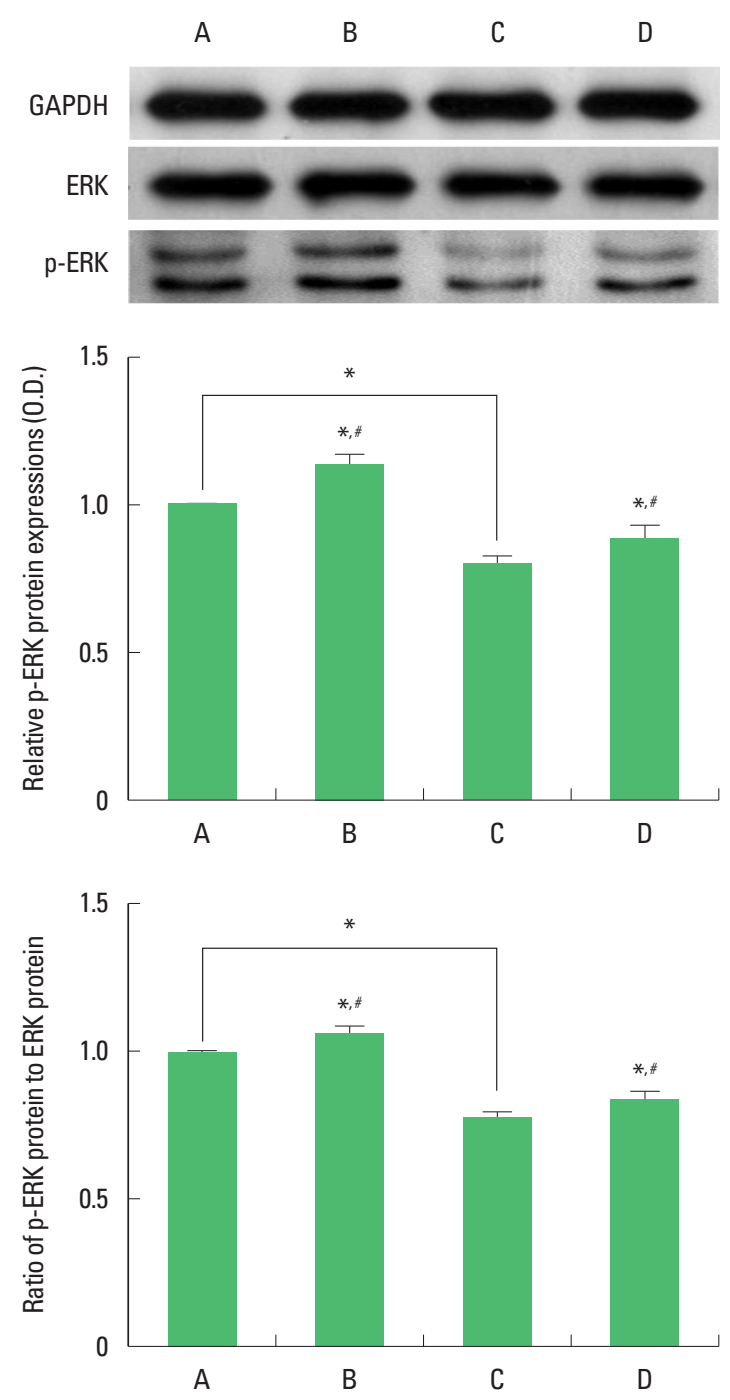

Fig. 4. Extracellular signal-regulated protein kinase (ERK) and phosphorylated-ERK (p-ERK) expression in the hippocampus. Upper panel: representative expression of ERK and p-ERK. Middle and lower panel: relative expressions of ERK and p-ERK. A, sham-operation group; $B$, sham-operation and exercise group; $C$, ischemia-induction group; $D$, ischemia-induction and exercise group. ${ }^{*} P<0.05$ compared to the sham-operation group. ${ }^{\sharp} P<0.05$ compared to the ischemia-induction group.

pression was decreased by ischemia induction, resulting in decrease of p-PI3-kinase/PI3-kinase ratio. Treadmill exercise increased p-PI3kinase expression in the ischemia gerbils, resulting in increase of p-PI3-kinase/PI3-kinase ratio.

\section{Expression of caspase-3 in the hippocampal CA1 region}

The results of the expression of caspase- 3 in the hippocampal CA1 region are presented in Fig. 7. Caspase-3 expression was decreased by ischemia induction. Treadmill exercise increased caspase-3
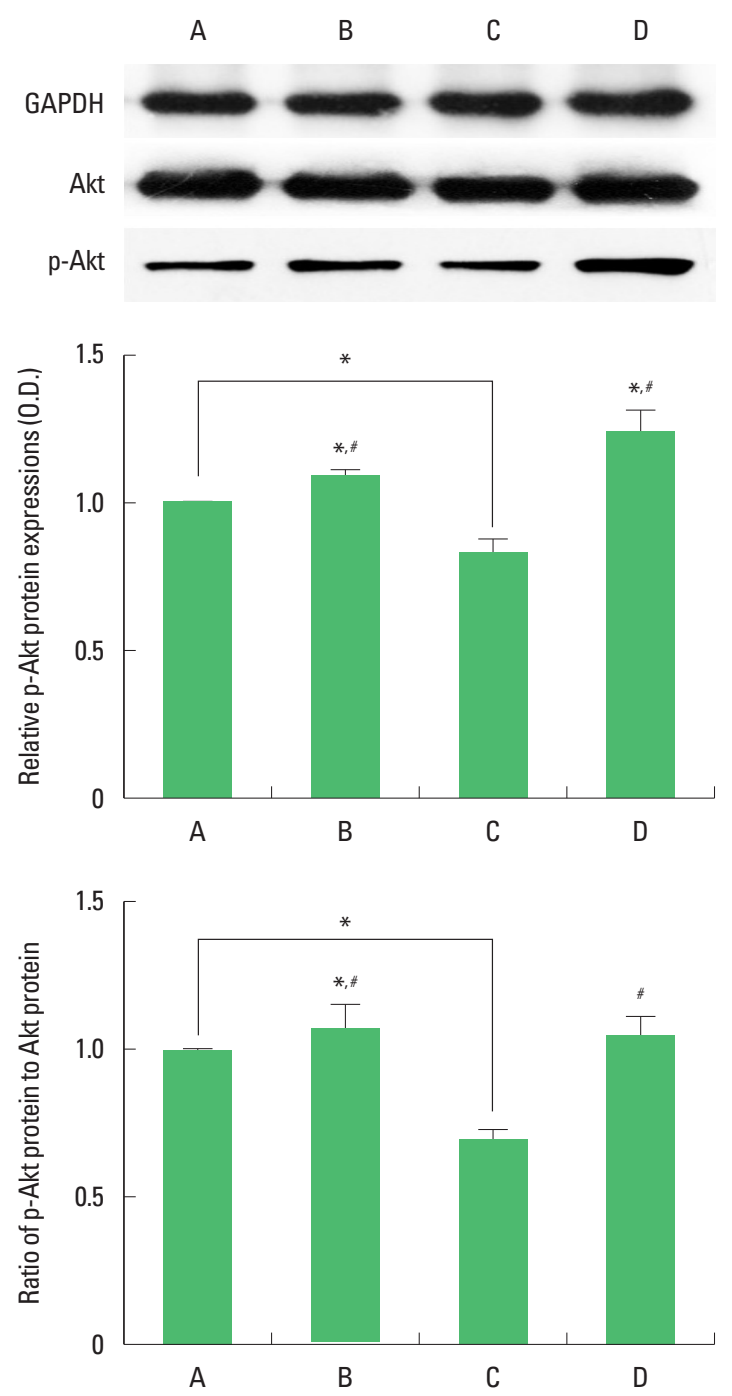

Fig. 5. Protein kinase B (Akt) and phosphorylated-Akt (p-Akt) expression in the hippocampus. Upper panel: representative expression of Akt and p-Akt. Middle and lower panel: relative expressions of Akt and p-Akt. A, sham-operation group; $B$, sham-operation and exercise group; $C$, ischemia-induction group; $D$, ischemia-induction and exercise group. ${ }^{*} P<0.05$ compared to the sham-operation group. ${ }^{\#} P<0.05$ compared to the ischemia-induction group.

expression in the ischemia gerbils.

\section{Expression of Bax and $\mathrm{Bcl}-2$ in the hippocampus}

The results of the relative expression of $\mathrm{Bax}$ and $\mathrm{Bcl}-2$ in the hippocampus are presented in Fig. 8. Bax expression was increased and $\mathrm{Bcl}-2$ expression was decreased by ischemia induction, resulting in increase of $\mathrm{Bax} / \mathrm{Bcl}-2$ ratio. Treadmill exercise decreased Bax expression and increased $\mathrm{Bcl}-2$ expression in the ischemia gerbils, resulting in decrease of $\mathrm{Bax} / \mathrm{Bcl}-2$ ratio. 

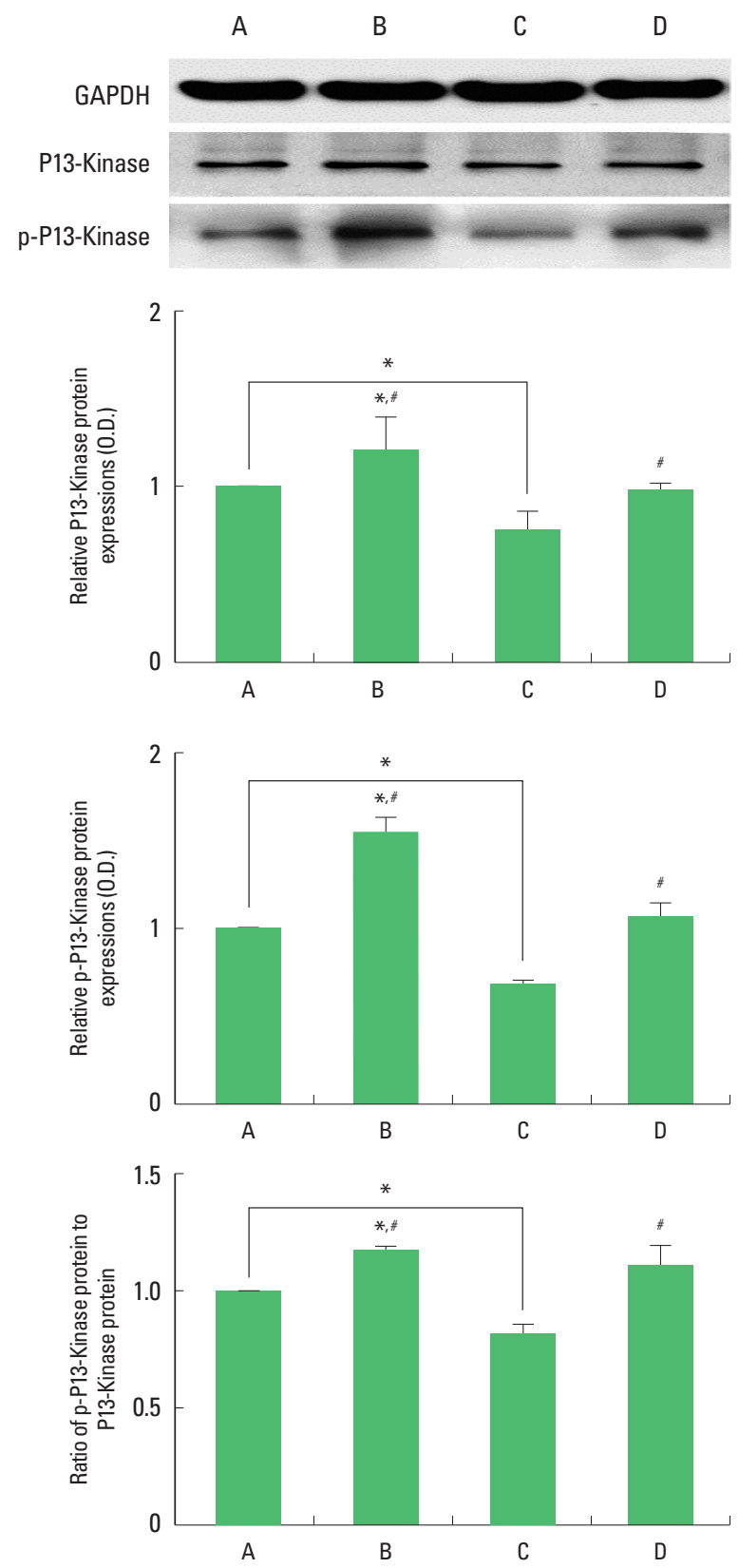

Fig. 6. Phosphatidylinositol 3-kinase (PI3-kinase) and phosphorylated PI3-kinase (p-PI3-kinase) expression in the hippocampus. Upper panel: representative expression of P|3-kinase and p-PI3-kinase. Middle and lower panel: relative expressions of PI3-kinase and p-PI3-kinase. A, sham-operation group; $\mathrm{B}$, sham-operation and exercise group; $C$, ischemia-induction group; $D$, ischemia-induction and exercise group. ${ }^{*} P<0.05$ compared to the sham-operation group. ${ }^{\#} P<0.05$ compared to the ischemia-induction group.

\section{DISCUSSION}

Hypoxic-ischemic brain damage deteriorated short-term memory in rats (Park et al., 2016) and ischemia impaired short-term
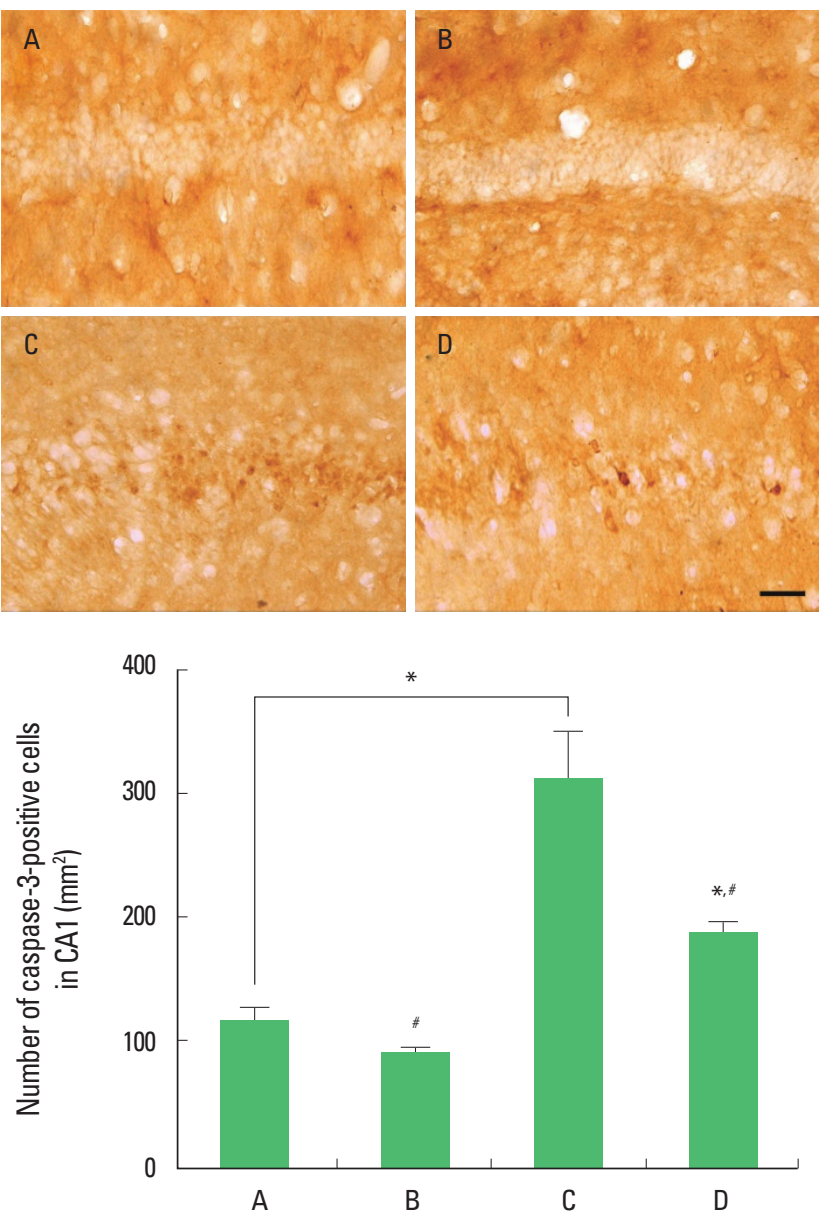

Fig. 7. Caspase-3 expression in the hippocampal CA1 region. Upper panel: photomicrographs of caspase-3-positive cells. The scale bar represents $50 \mu \mathrm{m}$. Lower panel: number of caspase-3-positive cells in each group. A, sham-operation group; $B$, sham-operation and exercise group; $C$, ischemia-induction group; $\mathrm{D}$, ischemia-induction and exercise group. ${ }^{*} P<0.05$ compared to the sham-operation group. ${ }^{\#} P<0.05$ compared to the ischemia-induction group.

memory in gerbils (Choi et al., 2017). Treadmill exercise improved memory function through suppressing apoptosis in the hippocampus following cerebral ischemia (Choi et al., 2017; Park et al., 2016). In this study, the latency in the step-down avoidance task was decreased by induction of ischemia, indicating that ischemia deteriorated short-term memory in gerbils. In contrast, treadmill exercise enhanced the latency in the step-down avoidance task.

BDNF exerts its neuroprotective effect against ischemic injury via activation of the ERK signaling pathway (Han and Holtzman, 2000). Furthermore, caspase- 3 is inhibited by increasing BDNF expression in cerebral ischemia (Cao et al., 2011; Choi et al., 2017). The protective effect of exercise on brain injury is due to the activation of BDNF signaling pathway by exercise (Ko et al., 2018). In the present results, BDNF and TrkB expression in the hippo- 

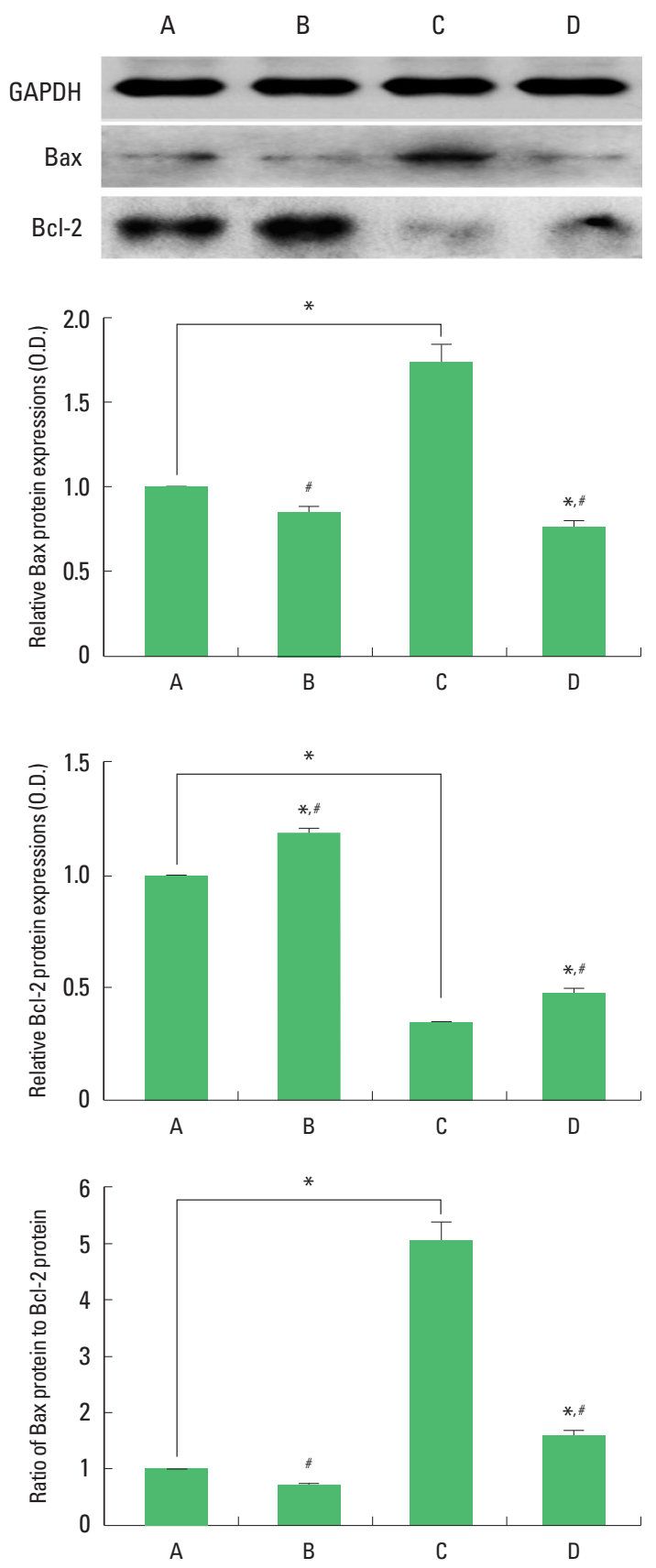

Fig. 8. Bax and Bcl-2 expression in the hippocampus. Upper panel: representative expression of $\mathrm{Bax}$ and $\mathrm{Bcl}-2$. Middle and lower panel: relative expressions of $\mathrm{Bax}, \mathrm{Bcl}-2$, and $\mathrm{Bax}$ to $\mathrm{Bcl}-2$ ratio. A, sham-operation group; $\mathrm{B}$, sham-operation and exercise group; $C$, ischemia-induction group; $D$, ischemia-induction and exercise group. ${ }^{*} P<0.05$ compared to the sham-operation group. ${ }^{*} P<0.05$ compared to the ischemia-induction group.

campus was suppressed by the induction of ischemia. In contrast, treadmill exercise enhanced BDNF and TrkB expression in the ischemia gerbils.

CREB-dependent expression of BDNF was increased by exer- cise (Tokuyama et al., 2000). CREB is an important transcription factor in the BDNF-TrkB signaling and its phosphorylation form of CREB (p-CREB) mediates neurogenesis in the adult hippocampal dentate gyrus after focal ischemia (Zhu et al., 2004). In the present study, induction of cerebral ischemia decreased phosphorylation of CREB in the hippocampus. In contrast, treadmill exercise increased phosphorylation of CREB in the hippocampus of ischemia gerbils.

PI3-kinase phosphorylates the 3'-hydroxyl group of phosphatidylinositols. This reaction leads to the activation of many intracellular signaling pathways that regulate functions as diverse as cell metabolism, survival, polarity, and vesicle trafficking (Knight et al., 2006). Akt acts downstream of PI3-kinase to regulate many biologic process such as proliferation, apoptosis, and growth. Akt plays an important role in cell survival signaling of the brain and it exerts a neuroprotective response to ischemia (Fukunaga and Kawano, 2003). Induction of global ischemia decreased Akt phosphorylation in the hippocampal CA1 (Koh et al., 2006). Neuroprotective role of the phosphorylation form of PI3-kinase/Akt pathway following cerebral ischemia is well documented (Carloni et al., 2010; Wang et al., 2009). Activated Akt inhibits apoptosis by phosphorylating its substrates, such as Bcl-2-associated death proteins (Hanada et al., 2004). Exercise-induced cognitive improvement is mediated by phosphorylation of PI3-kinase/Akt (Chae and Kim, 2009). In the present study, induction of cerebral ischemia suppressed phosphorylation of Akt and PI3-kinase in the hippocampus. In contrast, treadmill exercise enhanced phosphorylation of Akt and PI3-kinase in the hippocampus of ischemia gerbils.

ERK is involved in the regulating brain cell death and survival after ischemia (Irving and Bamford, 2002). Elevated ERK phosphorylation in the brain initiates a downstream signaling cascade, including CREB and BDNF, which initiates the positive feedback (Lonze and Ginty, 2002). The ERK-CREP-BDNF signaling cascade plays a crucial role in the learning and memory performance (Ying et al., 2002). In the present study, induction of cerebral ischemia decreased phosphorylation of ERK in the hippocampus. In contrast, treadmill exercise increased phosphorylation of ERK in the hippocampus of ischemia gerbils.

Apoptosis after cerebral ischemia is the final event that leads to the process of cell death (Mattson et al., 2001). Ischemia triggers apoptotic neurodegeneration through caspase-3 activation (Choi et al., 2017; Park et al., 2016). In the ischemic attack, increased expression of $\mathrm{Bcl}-2$ inhibited apoptosis, while over-expression of Bax promoted apoptosis (Choi et al., 2017; Park et al., 2016). Increase of Bax to Bcl-2 ratio represents apoptotic cell death (Song 
et al., 2018). In the present study, caspase- 3 expression in the hippocampal CA1 region was enhanced by the induction of cerebral ischemia insult. In contrast, treadmill exercise suppressed caspase-3 expression in the hippocampal CA1 region of ischemia gerbils. Bax expression was increased and $\mathrm{Bcl}-2$ expression was decreased in the hippocampus following cerebral ischemia. As a result, induction of cerebral ischemia increased the ratio of $\mathrm{Bax}$ to $\mathrm{Bcl}-2$. In contrast, treadmill exercise suppressed Bax expression and enhanced $\mathrm{Bcl}-2$ expression in the hippocampus of ischemia gerbils, resulting in decrease of $\mathrm{Bax}$ to $\mathrm{Bcl}-2$ ratio.

In this study, treadmill exercise improved short-term memory through increasing ERK and CREB phosphorylation with activation of Akt signaling with its downstream of BDNF-TrkB signaling in the cerebral ischemia gerbils. These results suggest that improvement of memory function after cerebral ischemia by treadmill exercise may be involved in the ERK-Akt-CREB-BDNF signaling pathway, resulting in inhibition of apoptosis in the hippocampus.

\section{CONFLICT OF INTEREST}

No potential conflict of interest relevant to this article was reported.

\section{ACKNOWLEDGMENTS}

This work was supported by the National Research Foundation of Korea Grant funded by the Korean Government (NRF-2018S1 A5A2A01031391).

\section{REFERENCES}

Cao Y, Mao X, Sun C, Zheng P, Gao J, Wang X, Min D, Sun H, Xie N, Cai J. Baicalin attenuates global cerebral ischemia/reperfusion injury in gerbils via anti-oxidative and anti-apoptotic pathways. Brain Res Bull 2011;85:396-402.

Carloni S, Girelli S, Scopa C, Buonocore G, Longini M, Balduini W. Activation of autophagy and Akt/CREB signaling play an equivalent role in the neuroprotective effect of rapamycin in neonatal hypoxia-ischemia. Autophagy 2010;6:366-377.

Chae $\mathrm{CH}$, Kim HT. Forced, moderate-intensity treadmill exercise suppresses apoptosis by increasing the level of NGF and stimulating phosphatidylinositol 3-kinase signaling in the hippocampus of induced aging rats. Neurochem Int 2009;55:208-213.

Choi IY, Hwang L, Jin JJ, Ko IG, Kim SE, Shin MS, Shin KM, Kim CJ, Park SW, Han JH, Yi JW. Dexmedetomidine alleviates cerebral ischemia-in- duced short-term memory impairment by inhibiting the expression of apoptosis-related molecules in the hippocampus of gerbils. Exp Ther Med 2017;13:107-116.

Fukunaga K, Kawano T. Akt is a molecular target for signal transduction therapy in brain ischemic insult. J Pharmacol Sci 2003;92:317-327.

Han BH, Holtzman DM. BDNF protects the neonatal brain from hypoxicischemic injury in vivo via the ERK pathway. J Neurosci 2000;20:57755781.

Hanada M, Feng J, Hemmings BA. Structure, regulation and function of PKB/AKT--a major therapeutic target. Biochim Biophys Acta 2004; 1697:3-16.

Hara T, Hamada J, Yano S, Morioka M, Kai Y, Ushio Y. CREB is required for acquisition of ischemic tolerance in gerbil hippocampal CA1 region. J Neurochem 2003;86:805-814.

Impey S, McCorkle SR, Cha-Molstad H, Dwyer JM, Yochum GS, Boss JM, McWeeney S, Dunn JJ, Mandel G, Goodman RH. Defining the CREB regulon: a genome-wide analysis of transcription factor regulatory regions. Cell 2004;119:1041-1054.

Irving EA, Bamford M. Role of mitogen- and stress-activated kinases in ischemic injury. J Cereb Blood Flow Metab 2002;22:631-647.

Kim M, Kim TW, Kim CJ, Shin MS, Hong M, Park HS, Park SS. Berberine ameliorates brain inflammation in poloxamer 407-induced hyperlipidemic rats. Int Neurourol J 2019;23(Suppl 2):S102-S110.

Knight ZA, Gonzalez B, Feldman ME, Zunder ER, Goldenberg DD, Williams O, Loewith R, Stokoe D, Balla A, Toth B, Balla T, Weiss WA, Williams RL, Shokat KM. A pharmacological map of the PI3-K family defines a role for p110alpha in insulin signaling. Cell 2006;125:733-747.

Ko IG, Kim CJ, Kim H. Treadmill exercise improves memory by up-regulating dopamine and down-regulating D2 dopamine receptor in traumatic brain injury rats. J Exerc Rehabil 2019;15:504-511.

Ko IG, Kim SE, Hwang L, Jin JJ, Kim CJ, Kim BK, Kim H. Late starting treadmill exercise improves spatial leaning ability through suppressing CREP/BDNF/TrkB signaling pathway following traumatic brain injury in rats. J Exerc Rehabil 2018;14:327-334.

Koh PO, Cho GJ, Choi WS. 17beta-estradiol pretreatment prevents the global ischemic injury-induced decrease of Akt activation and bad phosphorylation in gerbils. J Vet Med Sci 2006;68:1019-1022.

Lee JM, Baek SS, Kim TW, Park HS, Park SS, Park JM, Kim YJ, Lee HS, Shin MS. Preischemic treadmill exercise improves short-term memory by inhibiting hypoperfusion-induced disruption of blood-brain barrier after bilateral common carotid arteries occlusion. J Exerc Rehabil 2019; 15:370-376

Lonze BE, Ginty DD. Function and regulation of CREB family transcription factors in the nervous system. Neuron 2002;35:605-623.

Mattson MP, Duan W, Pedersen WA, Culmsee C. Neurodegenerative 
disorders and ischemic brain diseases. Apoptosis 2001;6:69-81.

Meller R, Minami M, Cameron JA, Impey S, Chen D, Lan JQ, Henshall DC, Simon RP. CREB-mediated Bcl-2 protein expression after ischemic preconditioning. J Cereb Blood Flow Metab 2005;25:234-246.

Numakawa T, Yokomaku D, Richards M, Hori H, Adachi N, Kunugi H. Functional interactions between steroid hormones and neurotrophin BDNF. World J Biol Chem 2010;1:133-143.

Park JH, Kim SE, Jin JJ, Choi HS, Kim CJ, Ko IG. Pentoxifylline alleviates perinatal hypoxic-ischemia-induced short-term memory impairment by suppressing apoptosis in the hippocampus of rat pups. Int Neurourol J 2016;20:107-113.

Park SS, Park HS, Jeong H, Kwak HB, No MH, Heo JW, Yoo SZ, Kim TW. Treadmill exercise ameliorates chemotherapy-induced muscle weakness and central fatigue by enhancing mitochondrial function and inhibiting apoptosis. Int Neurourol J 2019;23(Suppl 1):S32-S39.

Poo MM. Neurotrophins as synaptic modulators. Nat Rev Neurosci 2001; 2:24-32.

Santos AR, Comprido D, Duarte CB. Regulation of local translation at the synapse by BDNF. Prog Neurobiol 2010;92:505-516.

Song SH, Jee YS, Ko IG, Lee SW, Sim YJ, Kim DY, Lee SJ, Cho YS. Treadmill exercise and wheel exercise improve motor function by suppressing apoptotic neuronal cell death in brain inflammation rats. J Exerc Rehabil 2018;14:911-919.

Tokuyama W, Okuno H, Hashimoto T, Xin Li Y, Miyashita Y. BDNF upregulation during declarative memory formation in monkey inferior temporal cortex. Nat Neurosci 2000;3:1134-1142.

Wang HY, Wang GL, Yu YH, Wang Y. The role of phosphoinositide-3-kinase/Akt pathway in propofol-induced postconditioning against focal cerebral ischemia-reperfusion injury in rats. Brain Res 2009;1297:177184.

Whitfield TW Jr, Shi X, Sun WL, McGinty JF. The suppressive effect of an intra-prefrontal cortical infusion of BDNF on cocaine-seeking is Trk receptor and extracellular signal-regulated protein kinase mitogen-activated protein kinase dependent. Version 2. J Neurosci 2011;31:834842.

Yamada K, Mizuno M, Nabeshima T. Role for brain-derived neurotrophic factor in learning and memory. Life Sci 2002;70:735-744.

Yan XB, Hou HL, Wu LM, Liu J, Zhou JN. Lithium regulates hippocampal neurogenesis by ERK pathway and facilitates recovery of spatial learning and memory in rats after transient global cerebral ischemia. Neuropharmacology 2007;53:487-495.

Ying SW, Futter M, Rosenblum K, Webber MJ, Hunt SP, Bliss TV, Bramham CR. Brain-derived neurotrophic factor induces long-term potentiation in intact adult hippocampus: requirement for ERK activation coupled to CREB and upregulation of Arc synthesis. J Neurosci 2002; 22:1532-1540.

Zhu DY, Lau L, Liu SH, Wei JS, Lu YM. Activation of cAMP-response-element-binding protein (CREB) after focal cerebral ischemia stimulates neurogenesis in the adult dentate gyrus. Proc Natl Acad Sci U S A 2004; 101:9453-9457. 